\title{
Quality of life following acoustic neuroma surgery
}

\section{Marc S. Schwartz, M.D., Seth A. Riddle, M.D., Johnny B. Delashaw, Jr., M.D., Michael A. Horgan,} M.D., Jordi X. Kellogg, M.D., and Sean O. McMenomey, M.D.

Departments of Neurosurgery and Otolaryngology/Head and Neck Surgery, Oregon Health Sciences University, Portland, Oregon

In the treatment of acoustic neuroma, operative results have improved greatly during recent years, with high rates of functional cranial nerve preservation. Because of this, it has become more important to consider issues of patient satisfaction and quality of life (QOL) following treatment for these lesions. The authors have developed a novel questionnaire designed to measure QOL in patients with acoustic neuromas, and they administered it to 50 consecutive patients at least 6 months after acoustic neuroma surgery. Overall QOL was judged to be good but with definite minor difficulties, including some problems with hearing, facial nerve function, headache, tinnitus, dizziness, activity level, enjoyment of life, and emotional well-being. No significant differences were found between age groups and different operative approaches, and only minor differences were found in relation to tumor size. Patients with intracanalicular tumors fared no better than those with cerebellopontine angle tumors. Analysis of the data suggests an overall good outcome from acoustic neuroma surgery; however, when discussing the possible effects on postoperative QOL, even the potential minor problems should not be minimized, especially in patients undergoing operation for small or intracanalicular tumors.

\section{Key Words * acoustic neuroma * quality of life}

Acoustic neuromas are relatively common benign tumors that arise from Schwann cells of the vestibular nerve and are located at the cerebellopontine angle (CPA) or within the internal auditory canal (IAC), and they account for approximately $10 \%$ of all diagnosed intracranial tumors, with an incidence rate of 0.8 to $2.7 \%$ as demonstrated by postmortem studies.[2] More aggressive screening, together with improvements in audiological testing and the widespread use of magnetic resonance (MR) imaging, has resulted in a significantly increased rate of diagnosis of acoustic neuroma.[11]

Although pathologically benign, the location of these tumors may lead to progressive severe neurological dysfunction and death if growth remains unchecked. Historically, the major criteria for operative success in their treatment have been the prolongation of patient survival time and the avoidance of major complications.[14] In more recent years, the determination of successful treatment has also included functional preservation of the seventh and eighth cranial nerves, as measured by facial strength and useful hearing.[4-6,10] Although questions of specific neurological function may begin to address the 
patient's overall quality of life (QOL), various other factors, such as headache and dizziness as well as the ability to resume employment and full recreational activity, may affect a patient's QOL and psychosocial well-being. These questions have only rarely been addressed.[20]

Before the age of modern imaging and microsurgery, diagnosis was generally delayed until the development of brainstem dysfunction, and treatment was often deferred until the onset of acutely life threatening hydrocephalus. As the ability to make earlier diagnoses of these lesions has developed, QOL issues have become more important. Controversy exists regarding the recommended treatment course for patients in whom small acoustic neuromas have been diagnosed. Immediate operative intervention using several possible operative approaches, stereotactic radiosurgery, and deferral of treatment have all been advocated.[1,3,12,15,16] Proper evaluation of these options should include not only patient survival and complication rates but also the impact on overall QOL.

Relatively few studies have attempted to assess QOL in patients with acoustic neuroma. Wiegand and Fickel[20] designed a lengthy questionnaire with open-ended questions to determine how respondents fared following surgery for acoustic neuroma. An adaptation of this questionnaire was used to study outcomes of a more complete patient series in Denmark.[13] Further studies in Great Britain and The Netherlands were conducted using more quantifiable data.[9,19] However, all of these studies solicited responses concerning the absence or presence of various symptoms, requiring subjective interpretation to infer the impact on overall QOL.

The present study is based more directly on an instrument developed by Hassan and Weymuller[7] to evaluate QOL after treatment for head and neck cancer. This questionnaire was designed to determine not the presence of various symptoms or life issues but rather the effects that these symptoms or life issues have on the patient's ability to continue functioning normally and with emotional well-being. By directly asking patients about the perceived effects of operative sequelae on QOL, the examiner is released from the need to infer QOL from the presence of symptoms reported by patients.

\section{CLINICAL MATERIAL AND METHODS}

\section{Patient Population}

This retrospective study was based on responses to a telephone questionnaire administered by a single interviewer a minimum of 6 months following operative treatment (range 6-50 months). The study group included all patients who underwent acoustic neuroma surgery at Oregon Health Sciences University between 1991 and 1995. Of the 57 patients who underwent operation, seven could not be contacted, leaving 50 patients to comprise the study population. (During the same period, nine other patients were seen and underwent follow-up serial MR imaging, two patients were referred for stereotactic radiosurgery, and 15 patients underwent operations or were followed up at other institutions; these patients were not included in the study.) Note should be made that the annual number of operations rose sharply during the study period (Table 1). This likely represented not only an increase in diagnostic capabilities but also the formation of an otolaryngology-neurosurgery team that generated more referrals. 


\begin{tabular}{|cc|}
\hline \multicolumn{2}{|c|}{ TABLE 1 } \\
NUMBER OF PATIENTS WITH AN ACOUSTIC \\
NEUROMA TREATED AT OREGON HEALTH \\
SCIENCES UNIYERSTY OVER A 5-YEAR PER IOD \\
\hline \hline Year & No. of Cas es \\
\hline 1991 & 1 \\
1992 & 5 \\
1993 & 11 \\
1994 & 17 \\
1995 & 16 \\
\hline
\end{tabular}

Relevant clinical details were collected from patient records. Tumor size and location were determined from preoperative MR images and were grouped into four categories: 1) purely intracanalicular; 2) CPA, $<2 \mathrm{~cm}$; 3) CPA, 2 to $4 \mathrm{~cm}$; and 4) CPA, >= $4 \mathrm{~cm}$. Patient age at surgery, operative approach (suboccipital, translabyrinthine, middle fossa, or combined), and postoperative facial nerve function (House-Brackmann[8] grade; >= 6 month follow up) were also noted.

\section{Description and Administration of Questionnaire}

Our questionnaire, which was based on the QOL instrument developed by Hassan and Weymuller[7] was designed specifically to assess acoustic neuroma patients (Table 2). It consisted of nine questions, each of which described an aspect of dysfunction or limitation that would be potentially expected in patients undergoing treatment for acoustic neuroma. Each question had several possible answers, allowing respondents to describe their level of functioning and the effects of various problems on overall functional status. 
1) On the topic of pain $\&$ headache

100: I have no increase in the occurrence or severity of pain or headaches

75: I have mild pain or occasional headaches that do not require medication

50: I have moderate pain or headaches requiring regular medication

25: I have severe pain \& br headaches controlled onl y bynarcotics

0 : I have severe pain \& br headaches not controlled by narcotics

2) On the topic of facial ner 'e ele function

100: I am not bothered byany facial or eye meakness

75: I have mild facial or eye me akness that does not bo ther or limit me

50: I have facial $\&$ br eye meakness that limits or hinders me or requires frequent care

25: I have facial \&br eye meakness that has limited my work, social, or recreational pattern

0: I have facial \&br eye meakness that has caused difficulty whision, speaking, or eating

3) On the topic of hearing (operated ear)

100: I have adequate hearing whout a hearing aid

75: I have adequate hearing $w /$ the use of a hearing aid

50: I have poor hearing w/a hearing aid

25: I have little useful hearing

0 : I have no useful hearing

4) On the topic of tinnitus

100: I have less tinnitus than preoperatively

75: I have no change in tinnitus

50: I have slight y more tinnitus, but it is not bothersome

25: I am significantly bo thered by tinnitus

0 : I have tinnitus that lim its my activity or has required medication, treatment, or counseling

5) On the topic of 'ertigo or dizziness

100: I have no vertigo or dizziness

75: I have less vertigo or diziness than I did before surgery

50: I have vertigo or diziness about the same now as it was before surgery

25: I have vertigo or dizziness more severe since surgery'

0 : I am significantly limited by wertigo or dizziness

6) On the topic of activity le vel

100: I am comple tely independent in caring for m yself

75: I require occasional assistance w/a few selected activities

50: I require regular assistance w/a range of activities

25: I am unable to drive or malk inde pendently but am able to bathe, dress, \& feed m yself

0 : I require assistance $w /$ bathing, dressing or eating

7) On the topic of employment

100: I have a job that is better or the same as before surgery

75: I have a less strenuous job sinoe surgery, woluntarily

50: I am unable to perform work at the same le vel as before surgery

25: I am atle to work only occasionally

0 : I am unable to work

8) On the topic of enjoyment of life

100: There are no significant limitations or recreation

75: There are a few things I can't do but still get out \& enjoylife

50: There are many times I wish I could get out

25: I don't go out because of meakness, dizziness, hearing loss or other problems

0 : I am severelylimited in what I can do

9) On the topic of emotional mell-being

100: I am happy\& content whylife

75: I am happy but there are a few minor things about m $y$ life that I would like to change

50: I am getting by

25: I am down all the time \& regret having surgery

0 : I am very depressed

Each of the nine categories was given an overall value of 100 points, with the final maximum score totaling 900 points. Responses to individual questions were rated from 100 (equivalents to normal or no 
effect on QOL) to 0 (signifying maximum dysfunction with severe repercussions on QOL.) Intermediate answers were graded with values of 25,50 , or 75 points. Because the questionnaire was specifically developed to measure QOL in patients with acoustic neuroma, the majority of questions concerned problems specific to this pathological process. The remainder of the questions concerned more general issues that affect QOL.

\section{Statistical Analysis}

Statistical analyses were carried out using commercially available software (Statview v4.5; Abacus Concepts, Berkeley, CA). All analyses were carried out among discrete data sets, using two-tailed, unpaired t-tests.

\section{RESULTS}

The average age of the respondents at the time of surgery was 52.7 years (range 25-85 years). There were 25 men and 25 women. Twelve tumors were located wholly within the IAC, 17 tumors were smaller than $2 \mathrm{~cm}$ in maximum diameter, 15 were 2 to $4 \mathrm{~cm}$, and six were larger than $4 \mathrm{~cm}$. Twenty-four patients underwent operation via the suboccipital approach, 20 by means of a translabyrinthine exposure, one via a middle fossa approach, and five via a combined approach. The demographic breakdown of patients is presented in Table 3. Postoperative House-Brackmann grade averaged 1.9 (37 of 50 patients receiving Grades 1-2), and useful hearing was preserved in five of 16 patients (three of seven with IAC tumors and two of nine with CPA tumors). House-Brackmann grade was found to depend significantly on tumor size $(<2 \mathrm{~cm}$, mean $=1.3,>=2 \mathrm{~cm}$, mean $=2.8 ; \mathrm{p}<0.0002)$.

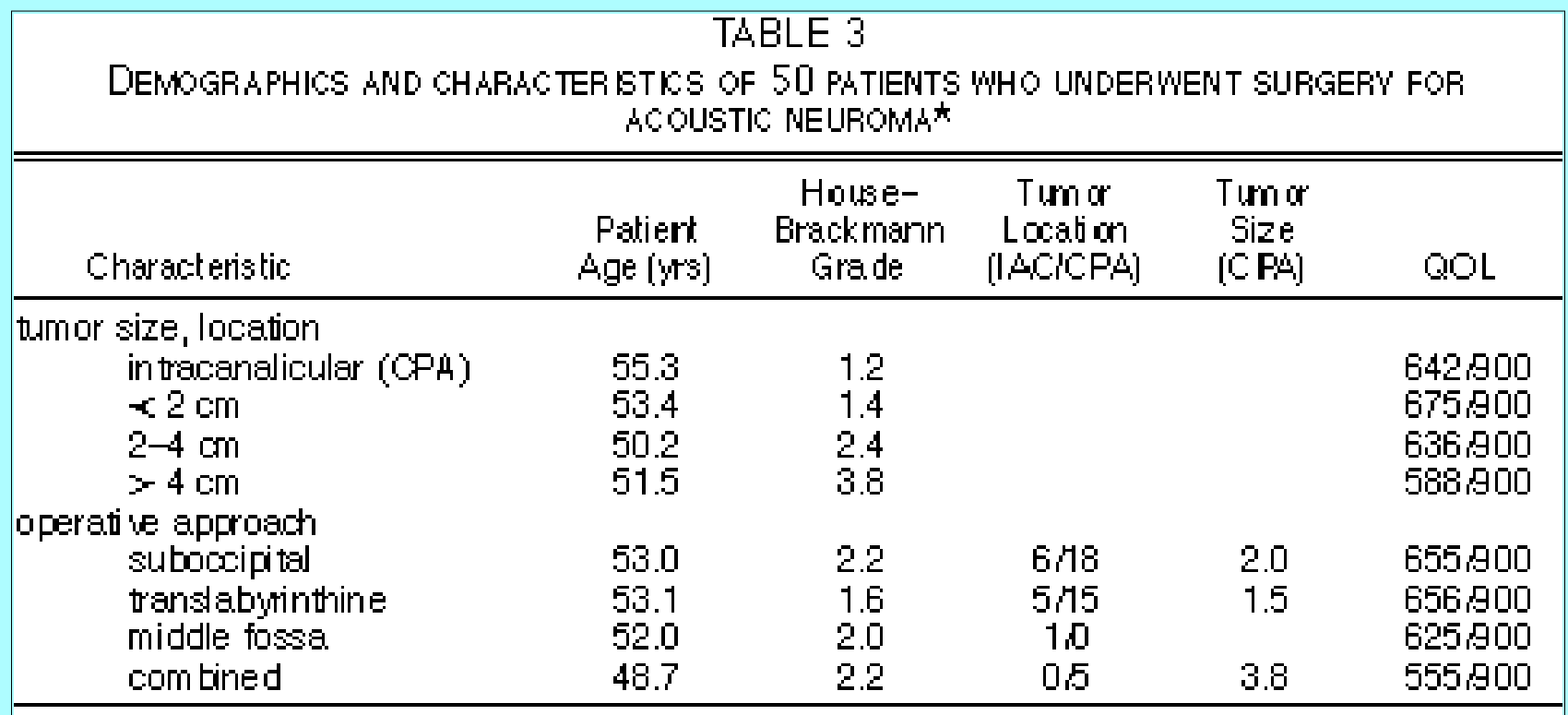

"Values for patient age, House-Erackmann grades, tumor size, and QOL are expressed as means.

Overall, patients scored an average of 645 of a possible 900 points on the acoustic neuroma QOL assessment instrument. Analysis was performed on data split by location (IAC compared with CPA), size $(<2 \mathrm{~cm}, 2-4 \mathrm{~cm},>=4 \mathrm{~cm})$, and operative approach (suboccipital compared with translabyrinthine). No significant differences were found between any of these groups; however, patients with larger tumors tended to score fewer points than those with smaller tumors. Interestingly, the patients with intracanalicular tumors averaged a lower score than those with CPA tumors, although this difference was not significant (Fig. 1). The data were also analyzed with regard to patient age and House-Brackmann grade, and no significant differences in overall scores were found (patients with House-Brackmann 
Grades 1-2 averaged 654 of 900 points and those with Grades 3-6 averaged 618 of $900 ; p=0.40$ ).

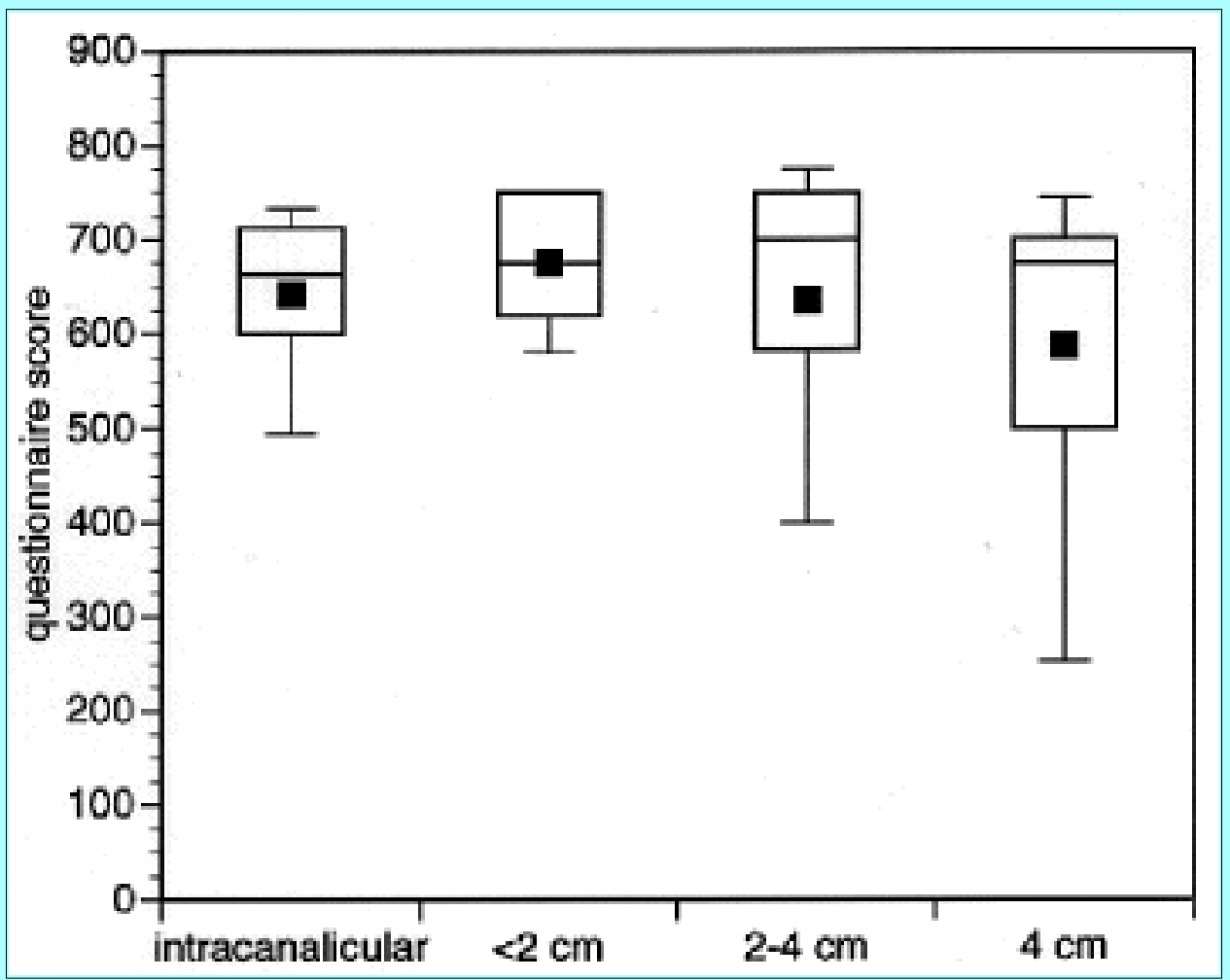

Fig. 1. Box plot displaying response to QOL questionnaire split by tumor size. The top, middle, and bottom of the boxes represent the 75th, 50th (median), and 25th percentiles, respectively. The mean responses are represented by small square symbols, and confidence intervals (at $\mathrm{p}=0.10$ ) are also shown.

When answers to individual questions were statistically analyzed, very few significant differences were found. Patients with intracanalicular tumors were more likely to be bothered by tinnitus than those with CPA tumors (61\% compared with $80 \%$ respectively; $\mathrm{p}=0.04)$. Significant differences between the suboccipital and translabyrinthine approaches were found only in regard to headache, with patients undergoing suboccipital procedures faring more poorly ( $71 \%$ compared with $86 \%$ respectively; $p=$ 0.03 ). Of course, significant differences in hearing were found among the various operative-approach groups, but this reflected often divergent goals in relation to hearing preservation.

\section{DISCUSSION}

Outcome after acoustic neuroma surgery has most often been measured in terms of the avoidance of serious complications and the preservation of specific neurological function.[4-6,10] Other than issues of operative mortality and serious morbidity rates, of course, the main determinants of operative success are preservation of function of the seventh and eighth cranial nerves. In the current study there were no deaths resulting from surgery, and postoperative seventh and eighth cranial nerve function was similar to that reported in previous studies. Excellent or good facial nerve function (House-Brackmann Grades 1-2) was attained in $74 \%$ of total cases and in $90 \%$ of cases with tumors smaller than $2 \mathrm{~cm}$ in diameter. Preservation of useful hearing was accomplished in $31 \%$ of cases in which it was attempted. This, however, represented only $10 \%$ of the entire series.

The issue of overall QOL measurement following acoustic neuroma surgery was first explored by Weigand and Fickel[20] in 1989 in an open-ended-question survey of Acoustic Neuroma Association 
members in which they found that patients were bothered not only by cranial nerve dysfunction but also by a variety of physical, psychological, and social problems, including depression, anxiety, headache, sleep disturbance, fatigue, dental problems, and speech or swallowing difficulties. As noted by the authors, however, the data were significantly skewed by the selection of a patient group involved with a self-help organization. Also, the use of open-ended questions, although appropriate for gathering inclusive information regarding potential problems, precluded accurate statistical analysis.

Several subsequent studies have also attempted to address similar issues. In these studies more closed-ended questions were used that eliminated the bias inherent in the initial study. Parving, et al.,[13] found similar postoperative problems with regard to headache, anxiety, depression, and other issues in a group of patients following translabyrinthine surgery. Irving, et al.,[9] used a questionnaire adapted from the European Organization for Research into the Treatment of Cancer core questionnaire and found that, despite a wide range of problems, most patients continued to function at a high level following acoustic neuroma surgery. They found a significant difference in functioning between patients with small tumors $(<1.5 \mathrm{~cm})$ and large tumors and, based on this, recommended surgery rather than observation in those patients with small tumors. Van Leeuwen, et al.,[19] found that QOL following acoustic neuroma surgery correlated highly with overall state of health as measured by Dutch Central Bureau of Statistics measurements and that overall state of health was lower for acoustic neuroma patients than for the general population. They found that in patients with smaller tumors $(<2.5 \mathrm{~cm})$, the translabyrinthine approach yielded better outcomes than did the suboccipital approach.

In all of these studies the authors attempted to measure changes in levels of functioning based on autobiographic measurements or relied on the interviewer's interpretation of answers to determine actual effects on overall QOL. That is, questions were in the form of, "My facial functioning is worse than it was preoperatively" or "I am significantly bothered by facial weakness." The current questionnaire, however, was based more directly on that developed by Hassan and Weymuller[7] to study QOL following treatment for head and neck cancers, and questions were designed to be nonautobiographic and to allow self-interpretation of effect on overall QOL; that is, "Facial or eye weakness has limited my performance at work or changed my social and/or recreational patterns."

Very few differences were found in overall QOL among subsets of the patients included in this study. Patient age and operative approach had no statistical effect on overall QOL, although patients who underwent operation via suboccipital approach were more likely to be bothered by headache. Based on other studies, tumor size should be expected to have had a significant effect.[17,18] Our results, however, did not reach significance among various subgroups. Most interesting, perhaps, is the lack of difference between patients with intracanalicular tumors and those with larger, CPA tumors. In fact, overall QOL was worse (although not significantly so) for patients with intracanalicular tumors than for those with small $(<2-\mathrm{cm})$ CPA tumors. There are several possible explanations for this. Symptoms at presentation were not rigorously studied and might account for this observation. However, the only specific difference seen in the intracanalicular group involved more difficulty with tinnitus, which has not been shown to be more problematic with intracanalicular tumors. Increased problems with vertigo were not demonstrated.

Another more difficult problem to assess might be one of patient expectations. If patients were told they had large brain tumors, or even smaller but serious CPA tumors that required resection, postoperative problems might be taken in stride by many patients. If, however, they were told that they had small tumors not affecting the brain that might be extirpated before causing real harm, the patients might be more sensitive to any nonideal results. This idea is further supported by the findings in relation to 
House-Brackmann grade, which is an objective measure and correlates strongly with tumor size. Despite the severe problems that might be expected with poor facial nerve function, no significant difference in overall QOL was found between patients with fair or poor facial nerve function compared to those with excellent or good function. Because most patients with fair or poor facial nerve function had larger tumors, they may have been more able to reconcile their facial weakness, without perceiving it as a major QOL problem.

It is difficult to draw conclusions from this study with regard to the optimum treatment strategy for patients with acoustic neuroma. The average total score of 645 of 900 points (71\%) placed patients in the range of mild, but definite, limitations on QOL. These results are quite satisfactory and in line with the results of previous studies. However, any firm statements regarding treatment options would have to be based on not only preoperative QOL data, but also on data about patients undergoing alternative modes of treatment such as radiosurgery and observation with serial MR imaging. A prudent conclusion from this study might be that patients' expectations should be considered when predicting postoperative QOL. Assuming that surgical treatment of larger tumors is more imperative with regard to preservation of life and avoidance of catastrophic morbidity, these results suggest that it is precisely the small and intracanalicular tumors that should not be taken lightly when patients are prepared for surgery and potential postoperative problems are discussed.

Although the retrospective nature of this study and the lack of preoperative data significantly limit its utility in the operative decision-making process, it does more directly address the issue of patients' perceived QOL than previous reports. These data are especially useful in regard to issues such as patient satisfaction. A prospective study in which a questionnaire similar to the one in this study and in which results are compared over time beginning in the preoperative phase is underway and will provide more direct information on the adequacy of treatment and the relative success of various types of treatment with regard to perceived QOL.

\section{CONCLUSIONS}

Patients' expectations should be considered when predicting postoperative QOL in the treatment of acoustic neuroma. Despite better postoperative outcomes by traditional measures, patients with smaller tumors do not report better postoperative QOL than those with larger tumors. Our results suggest that when patients with small and intracanalicular tumors are prepared for surgery, the potential for postoperative problems, including even those that may be considered "minor," such as headache, dizziness, or tinnitus, should not be underemphasized.

$\underline{\text { Click here to view commentary on this article. }}$

\section{References}

1. Bederson JB, von Ammon K, Wichmann WW, et al: Conservative treatment of patients with acoustic tumors. Neurosurgery 28:646-651, 1991

2. Brackmann DE, Green JD Jr: Cerebellopontine angle tumors, in Bailey BJ, Johnson JT, Kohut RI, et al (eds):Head and Neck Surgery-Otolaryngology. Philadelphia: JB Lippincott, 1993, Vol 2, pp1803-1819

3. Cox GJ: Intracanalicular acoustic neuromas: a conservative approach. Clin Otolaryngol 18:153-154, 1993 
4. Glasscock ME, Kvelton JF, Jackson CG: A systematic approach to the surgical management of acoustic neuroma. Laryngoscope 96:1088-1094, 1986

5. Hardy DG, Macfarlane R, Baguley D, et al: Surgery for acoustic neurinoma: an analysis of 100 translabyrinthine operations. J Neurosurg 71:799-804, 1989

6. Harner SG, Laws ER Jr: Posterior fossa approach for removal of acoustic neurinomas - recent experience. Arch Otolaryngol 107:590-593, 1981

7. Hassan SJ, Weymuller EA Jr: Assessment of quality of life in head and neck cancer patients. Head Neck 15:485-496, 1993

8. House JW, Brackmann DE: Facial nerve grading system. Otolaryngol Head Neck Surg 93:146-147, 1985

9. Irving RM, Beynon GJ, Viani L, et al: The patient's perspective after vestibular schwannoma removal: quality of life and implications for management. Am J Otol 16:331-337, 1995

10. King TT, Morrison AW: Translabyrinthine and transtentorial removal of acoustic nerve tumors. Results in 150 cases. J Neurosurg 52:210-216, 1980

11. Moffat DA, Hardy DG, Baguley DM: Strategy and benefits of acoustic neuroma searching. J Laryngol Otol 103:51-59, 1989

12. Nedzelski JM, Canter RJ, Kassel EE, et al: Is no treatment good treatment in the management of acoustic neuromas in the elderly? Laryngoscope 96:825-829, 1986

13. Parving A, Tos M, Thomsen J, et al: Some aspects of life quality after surgery for acoustic neuroma. Arch Otolaryngol Head Neck Surg 118:1061-1064, 1992

14. Pennybacker JB, Cairns H: Results in 130 cases of acoustic neuroma. J Neurol Neurosurg Psychiatry 13:272-277, 1950

15. Ramsay HAW, Luxford WM: Treatment of acoustic tumours in elderly patients: is surgery warranted? J Laryngol Otol 107:295-297, 1993

16. Ramsden RT, Moffat DA: Intracanalicular acoustic neuromas: the case for early surgery. Clin Otolaryngol 19:1-2, 1994

17. Samii M, Matthies C, Tatagiba M: Intracanalicular acoustic neurinomas. Neurosurgery 29:189-199, 1991

18. Schwaber MK: Acoustic neuromas and tumors of the cerebellopontine angle, in Glasscock ME III, Shambaugh GE (eds): Surgery of the Ear, ed 4. Philadelphia: WB Saunders, 1990, pp 534-570

19. van Leeuwen JPPM, Braspenning JCC, Meijer H, et al: Quality of life after acoustic neuroma surgery. Ann Otol Rhinol Laryngol 105:423-430, 1996

20. Wiegand DA, Fickel V: Acoustic neuroma--the patient's perspective: subjective assessment of symptoms, diagnosis, therapy, and outcome in 541 patients. Laryngoscope 99:179-187, 1989 
Manuscript received June 12, 1998.

Accepted in final form August 14, 1998.

Address reprint requests to:Marc S. Schwartz, M.D., Division of Neurosurgery, MC-61, Albany Medical College, Albany, New York 12208-3479. 